\title{
Composição Corporal e Exigências Nutricionais em Cálcio e Fósforo para Ganho e Mantença de Cordeiros Santa Inês dos 15 kg aos 25 kg de Peso Vivo ${ }^{1}$

\author{
Luciana Castro Geraseev², Juan Ramón Olalquiaga Perez ${ }^{3}$, Kleber Tomás de Resende ${ }^{4}$, José \\ Cleto da Silva Filho ${ }^{3}$, Sarita Bonagurio ${ }^{2}$
}

\begin{abstract}
RESUMO - Este trabalho foi conduzido para determinar a composição corporal e estimar as exigências de cálcio e fósforo de cordeiros da raça Santa Inês. Foram utilizados 18 cordeiros machos inteiros, com peso médio inicial de $15 \mathrm{~kg}$, divididos em três grupos: seis animais abatidos no início do experimento, para avaliar o conteúdo de cálcio e fósforo corporal, servindo como animais referência para o método do abate comparativo; seis animais alimentados ad libitum; e seis com alimentação restrita (em nível de mantença $+20 \%$ ). Os cordeiros que receberam dietas ad libitum e restritas entraram no experimento aos pares e foram abatidos concomitantemente, quando o primeiro atingiu $25 \mathrm{~kg}$ de peso vivo. A composição corporal foi estimada a partir de equações de regressão do logaritmo da quantidade de cálcio e fósforo presentes no corpo vazio, em função do logaritmo do peso corporal vazio. As exigências líquidas de mantença e o coeficiente de absorção destes minerais foram determinados a partir da correlação entre a quantidade de mineral ingerida e a quantidade retida no corpo, enquanto as exigências líquidas para o ganho em peso foram estimadas a partir da derivação de equações de predição da composição corporal. As exigências líquidas de mantença, para animais entre 15 e $25 \mathrm{~kg}$ de peso vivo, foram $305 \mathrm{mg}$ de Ca/dia e $325 \mathrm{mg}$ de P/dia e as exigências líquidas por $\mathrm{kg}$ de ganho de peso vivo, para animais com 15 e $25 \mathrm{~kg}$ de peso vivo, 11,41 e 10,33 g Ca e 5,72 e 4,94 g P, respectivamente. Os coeficientes de absorção encontrados neste trabalho foram 0,44 e 0,55 para Ca e $\mathrm{P}$, respectivamente.
\end{abstract}

Palavras-chave: cálcio, composição corporal, exigências nutricionais, fósforo, ovinos, Santa Inês

\section{Body Composition and Requirements for Calcium and Phosphorus for Gain and Maintenance of Santa Ines Lambs from 15 to $25 \mathrm{~kg}$ of Body Weight}

\begin{abstract}
The research was carried out to determine body composition and calcium and phosphorus requirements of Santa Ines lambs. Eighteen entire male lambs with average initial live weight of $15 \mathrm{~kg}$ were used. The animals were allotted to three groups: six animals were slaughtered at the beginning of the experiment, to access the amount of calcium and phosphorus present in the body, as reference animals for the comparative slaughter technique. Six animals were ad libitum fed and six were restrict fed (maintenance level plus $20 \%$ ). The animals ad libitum and restrict fed started the experimental period by pairs and they were both slaughtered when the first reached $25 \mathrm{~kg}$ body weight. The body composition was estimated through the prediction equations obtained by regression of the logarithm of the amount of calcium and phosphorus in the empty body on the logarithm of the empty body weight. Net requirements for calcium and phosphorus for maintenance and the absorption coefficient were obtained through the correlation between the amount of each mineral consumed and retained in the animal body. The net requirements maintenance for live weight gain were obtained by means of derivation of the prediction of body composition equations. The net requirements maintainance of calcium and phosphorus for animals from 15 to $25 \mathrm{~kg}$ body weight were: $305 \mathrm{mg} \mathrm{Ca} /$ day and $325 \mathrm{mg} \mathrm{P} /$ day and net requirements for kg of the body weight gain for animals with 15 and $25 \mathrm{~kg} \mathrm{LW}$ were 11.41 and 10.33 $\mathrm{g} \mathrm{Ca}$ and 5.72 and $4.94 \mathrm{~g} \mathrm{P}$, respectively. The absorption coefficients were estimated to be .44 and 0.55 for Ca and P, respectively.
\end{abstract}

Key Words: body composition, calcium, nutritional requirements, phosphorus, lambs, Santa Inês

\section{Introdução}

As concentrações de cálcio e fósforo corporais são reflexo, principalmente, da proporção de ossos e gordura da carcaça; portanto, fatores como idade, raça, grupo genético, sexo, manejo alimentar e condições climáticas a que o animal são submetidos influem na concentração desses minerais.
O AGRICULTURAL RESEARCH COUNCIL ARC (1980) adotou valor médio de $11 \mathrm{~g} / \mathrm{kg}$ de peso corporal vazio (PCV), para o conteúdo corporal de cálcio, para ovinos em crescimento, considerando que a concentração de cálcio no ganho de peso é independente do peso do animal. ANNENKOV (1982) e GRACE (1983) encontraram valores médios, para cordeiros em crescimento, de 10,20 e 10,50 g de

\footnotetext{
${ }^{1}$ Extraído da Dissertação de Mestrado apresentada pelo primeiro autor à UFLA.

2 Aluno do Curso de Pós-Graduação em Zootecnia da UFLA - CEP 37200-000 - Lavras, MG. E.mail: Igerasev@ufla.br

3 Professor da Univ. Fed. de Lavras - CEP 37200-000 - Lavras - MG. E.mail: jroperez@ufla.br

${ }^{4}$ Professor da Univ. Estadual Paulista Campus de Jaboticabal - CEP 14870-000 - Jaboticabal, SP. E.mail: klebertr@fcav.unesp.br
} 
cálcio/kg de ganho de peso corporal, respectivamente. Segundo o ARC (1980), o conteúdo corporal de fósforo em ovinos em crescimento é de $6 \mathrm{~g} / \mathrm{kg}$ PCV e sua deposição no ganho de peso corporal vazio é constante. ANNENKOV (1982) e GRACE (1983) encontraram valores próximos aos recomendados pelo ARC (1980), considerando valor médio de 5,40 e 5,20 g P $/ \mathrm{kg}$ de ganho em peso, respectivamente.

O AGRICULTURAL AND FOOD RESEARCH COUNCIL - AFRC (1991) estima que os ossos contêm 50 a 100 g P/kg; os músculos, 2 a 3 g de P/kg; e a gordura, quantidades mínimas na forma de fosfolipídeos. GRACE (1983), estudando a distribuição de diversos minerais em vários órgãos e tecidos de cinqüenta ovinos Romney, encontrou valores próximos aos do AFRC (1991), 1,30; 52,70; 0,30; e 0,16 g P/kg em músculos, ossos, pele e lã, respectivamente.

As exigências de cálcio e fósforo dos animais dependem de vários fatores: peso corporal, velocidade de crescimento, idade, nível de produção e gestação. Para que estes minerais sejam assimilados pelo organismo animal, é necessário o fornecimento de nível adequado de ambos minerais na dieta, pois o excesso ou deficiência de um interfere na própria utilização do outro (CAVALHEIRO e TRINDADE, 1992). O ARC (1980) e o NATIONAL RESEARCH COUNCIL - NRC (1985) admitem que os requerimentos líquidos de macroelementos minerais são constantes e independem do peso do animal. Nas recomendações das exigências minerais, o NRC (1985) considerou requerimento absoluto de cálcio e fósforo, para cordeiros em crescimento, de $183 \mathrm{mg}$ Ca e $103 \mathrm{mg} \mathrm{P} / \mathrm{kg}$ de peso vivo/dia, enquanto o ARC (1980) estimou este requerimento como $11 \mathrm{~g} \mathrm{Ca}$ e 6 g P/kg de PCV. Já o AFRC (1991) adotou equações com base no crescimento ósseo para estimar as exigências de cálcio e fósforo e considerou que a deposição destes elementos no corpo decresce à medida que o animal se torna adulto.

ANNENKOV (1982) apresentou uma tabela de exigências de cálcio e fósforo para várias categorias. Para animais com $10 \mathrm{~kg}$ de peso vivo e ganho diário de $100 \mathrm{~g}$, recomenda-se ingestão de $1,70 \mathrm{~g} \mathrm{Ca}$ e $1,10 \mathrm{~g}$ $\mathrm{P} /$ dia e, para ganho de $200 \mathrm{~g}, 3,30 \mathrm{~g}$ Ca e 1,90 g P/dia. Para animais com $20 \mathrm{~kg}$ de peso vivo e ganho diário de $100 \mathrm{~g}$, a recomendação é de 3,00 g Ca e 1,30 g P/dia e, para ganho diário de 200 g, 5,00 g Ca e 2,00 g P/dia.

Segundo BOIN (1985), em função das diferenças entre os sistemas de produção, as raças, os alimentos e as condições climáticas, é importante a realização de trabalhos com o objetivo de verificar as exigências minerais, sendo que este tipo de experimento deve ser feito regionalmente com os animais e nas condições dos sistemas de produção usados. No Brasil, os cálculos de rações baseiam-se em valores propostos pelo ARC (1980), NRC (1985) e AFRC (1991), os quais são obtidos em ambientes diferentes dos encontrados no Brasil e, muitas vezes, extrapolados de dados obtidos com outras espécies animais; por comseguinte, é fundamental o conhecimento das exigências nutricionais de macrominerais em condições brasileiras, com as raças existentes e adaptadas ao nosso ambiente.

Os objetivos deste trabalho foram determinar a composição corporal e estimar as exigências de cálcio e fósforo em cordeiros Santa Inês em crescimento, criados nas condições do Sul de Minas Gerais.

\section{Material e Métodos}

O experimento foi desenvolvido no Setor de Ovinocultura do Departamento de Zootecnia da Universidade Federal de Lavras, no período de setembro de 1997 a março de 1998. Foram utilizados 18 cordeiros machos inteiros da raça Santa Inês, com peso vivo médio inicial de $15 \mathrm{~kg}$. Após a desmama, aos 40 dias de idade, os animais foram confinados em grupo até atingirem o peso inicial para entrarem no experimento. Durante esse período, receberam alimentação à vontade, a qual possuía a mesma constituição da dieta experimental (Tabela 1).

Ao atingirem o peso inicial, seis animais escolhidos ao acaso foram abatidos servindo como animais referência; os demais entraram no experimento aos pares, sendo um para alimentação ad libitum e o outro para alimentação restrita. Os animais da alimentação ad libitum receberam quantidades de alimento que permitiram sobra de $20 \%$ do total oferecido, enquanto os animais do grupo de alimentação restrita receberam quantidades para atender ingestão diária de energia metabolizável, correspondendo à exigência de mantença, com acréscimo de $20 \%$, segundo as recomendações do ARC (1980).

$\mathrm{O}$ animal da alimentação ad libitum, assim que atingiu o peso pré-determinado para o abate $(25 \mathrm{~kg})$, foi abatido juntamente com o seu par da alimentação restrita. Previamente, os animais foram submetidos a jejum de 16 horas, com acesso à água. $\mathrm{O}$ abate foi feito por sangramento por intermédio do corte da carótida e jugular dos animais, sendo o sangue coletado, pesado e congelado para análises posteriores. Após a coleta do sangue, o conteúdo do trato digestivo, da bexiga e vesícula biliar foi eliminado para determinação 
Tabela 1 - Composição da dieta experimental, (\%MS)

Table 1 - Composition of the experimental diet (\%DM)

\begin{tabular}{|c|c|c|c|c|c|}
\hline $\begin{array}{l}\text { Ingrediente } \\
\text { Ingredient }\end{array}$ & $\begin{array}{l}\text { MS } \\
D M \\
(\%) \\
\end{array}$ & $\begin{array}{c}\mathrm{EM}^{1} \\
E M \\
(\mathrm{kcal} / \mathrm{kg})\end{array}$ & $\begin{array}{l}\mathrm{PB} \\
C P \\
(\%) \\
\end{array}$ & $\begin{array}{l}\mathrm{Ca} \\
(\%) \\
\end{array}$ & $\begin{array}{c}\mathrm{P} \\
(\%) \\
\end{array}$ \\
\hline $\begin{array}{l}\text { Milho } \\
\text { Corn }\end{array}$ & 66,23 & 2,087 & 6,49 & 0,022 & 0,196 \\
\hline $\begin{array}{l}\text { Farelo de soja } \\
\text { Soybean meal }\end{array}$ & 12,37 & 0,394 & 6,28 & 0,053 & 0,097 \\
\hline $\begin{array}{l}\text { Feno coast-cross } \\
\text { Coast-cross hay }\end{array}$ & 20,25 & 0,395 & 2,44 & 0,120 & 0,080 \\
\hline $\begin{array}{l}\text { Calcário } \\
\text { Limestone }\end{array}$ & 0,85 & - & - & 0,306 & - \\
\hline $\begin{array}{l}\text { Sal } \\
\text { Salt }\end{array}$ & 0,25 & - & - & - & - \\
\hline $\begin{array}{l}\text { Sup. mineral }{ }^{2} \\
\text { Mineral supplement }\end{array}$ & 0,01 & - & - & - & - \\
\hline $\begin{array}{l}\text { Sup. vitamínico }{ }^{2} \\
\text { Vitamin supplement }\end{array}$ & 0,04 & - & - & - & - \\
\hline Total & 100,00 & 2,876 & 15,21 & 0,501 & 0,373 \\
\hline
\end{tabular}

do peso do corpo vazio. O corpo do animal (subdividido em partes menores), juntamente com aparelho digestivo, vísceras, sangue, cabeça, patas e pele, foi acondicionado em sacos plásticos e congelados.

Para retirada das amostras para as análises químicas, o material congelado foi cortado em uma serra de fita e moído em cutter de 30 HP e 1775 rpm, sendo, em seguida, homogeneizado e congelado. Este procedimento foi repetido duas vezes antes da retirada das amostras para as análises químicas. O abate dos animais do grupo de referência e o processamento das amostras referentes ao corpo destes animais seguiram o mesmo procedimento do grupo ad libitum.

As análises químicas foram efetuadas segundo a metodologia descrita por SILVA (1981). As análises para determinação dos macrominerais nas amostras dos ingredientes da dieta, na dieta e na matéria seca desengordurada do corpo do animal, foram efetuadas por meio da digestão ácida com ácido nítrico e ácido perclórico, obtendo-se, dessa forma, a solução mineral, a partir da qual foram feitas diluições para determinação do cálcio e fósforo.

O fósforo foi determinado por redução do complexo fósforo-molibdato com ácido ascórbico e as leituras foram tomadas em colorímetro. Para o teor de cálcio, as diluições foram feitas adicionando-se cloreto de estrôncio e as leituras tomadas em espectrofotômetro de absorção atômica.

Para predição do conteúdo de cálcio e fósforo por quilo de peso corporal vazio dos animais, adotouse o modelo exponencial $\mathrm{y}=\mathrm{ax}{ }^{\mathrm{b}}$, preconizado pelo ARC (1980). Este modelo foi logaritmizado conforme a equação que se segue:

em que

$$
\log y=a+b \log x
$$

$\log \mathrm{y}=\log$ aritmo do conteúdo total do macromineral no corpo vazio;

a = intercepto;

$\log \mathrm{x}=\log$ aritmo do peso corporal vazio; $\mathrm{e}$

b = coeficiente de regressão do conteúdo do macromineral em função do peso corporal vazio.

Para composição do ganho de peso em cálcio e fósforo, utilizou-se a técnica do abate comparativo descrita pela ARC (1980), que possibilita a determinação da quantidade de mineral retido no corpo pela diferença entre a quantidade presente nos animais sacrificados em pesos diferentes, 15 e $25 \mathrm{~kg}$ de peso vivo.

As exigências líquidas para o ganho em peso corporal vazio de cálcio e fósforo foram estimadas a partir da derivação das equações de regressão citadas anteriormente, obtendo-se equações do tipo $y^{\prime}=\mathrm{b} 10^{-\mathrm{a}}$ $\mathrm{PCV}^{(\mathrm{b}-1)}$. As exigências líquidas desses minerais para o ganho de peso vivo foram obtidas por meio da conversão do peso corporal vazio em peso vivo, utilizando-se o fator 1,10 obtido pelo quociente $\mathrm{PV} / \mathrm{PCV}$ dos animais. Os requerimentos dietéticos foram então estimados aplicando-se o método fatorial, segundo a metodologia recomendada pelo ARC (1980). 
O coeficiente de absorção dos minerais foi obtido a partir da análise de regressão da quantidade retida no corpo vazio, em função da quantidade de cada mineral consumida pelos animais. A exigência líquida para mantença foi obtida por intermédio da extrapolação desta equação para o nível zero de ingestão.

\section{Resultados e Discussão}

Na Tabela 2, são apresentados os resultados médios e seus respectivos desvios-padrão da composição corporal em cálcio e fósforo e peso dos animais estudados. Observa-se aumento na concentração de matéria seca e gordura corporal, em função do aumento do peso vivo. Essa relação entre a concentração de gordura, matéria seca corporal e peso corporal foi observada por vários autores (BURTON e REID, 1969; RATTRAY et al., 1973; e SEARLE et al., 1979). Este fato justifica-se pela modificação da composição do ganho em peso com o aumento em peso dos animais.

A concentração de matéria seca (MS) encontrada neste trabalho foi de 32,36 e $35,91 \%$ do PCV e a de gordura, 10,02 e 14,43\% do PCV, para animais sem restrição alimentar com peso corporal vazio de 12,90 e $21,91 \mathrm{~kg}$, respectivamente. Estes valores foram menores que os obtidos por SEARLE et al. (1979) e BURTON et al. (1974) e maiores que os propostos por SEARLE et al. (1982) e KELLAWAY (1973). Por outro lado, estão próximos aos valores de concentração de gordura estimados pelo ARC (1980), cuja concentração foi 8,40 e 13,90\% do PCV, para cordeiros inteiros e não-merinos, com 15 e $25 \mathrm{~kg}$ de $\mathrm{PCV}$, respectivamente. Essas diferenças, principalmente nos valores de concentração de gordura, podem ser atribuídas a diferenças existentes entre as raças dos animais estudados, sexo, manejo alimentar e condições climáticas.

Quanto à concentração corporal de cálcio e fósforo para animais que não sofreram restrição alimentar, os valores obtidos foram 1,52 e $1,40 \% \mathrm{Ca}$ e $0,86 \mathrm{e}$ $0,75 \% \mathrm{P}$, para animais com peso corporal vazio de 12,90 e $21,91 \mathrm{~kg}$, respectivamente. Os dados obtidos neste experimento demonstraram redução na concentração de cálcio e fósforo no corpo vazio dos animais, em função do aumento do peso vivo dos mesmos.

Este decréscimo na concentração de cálcio e fósforo pode ser explicado pelo aumento na concentração de gordura nos animais mais pesados e pela redução no crescimento ósseo, à medida que aumenta o peso corporal, pois a maior parte do cálcio e fósforo corporal está presente neste tecido.
Segundo NOUR e THONNEY (1987), existe relação inversa entre as concentrações minerais nos tecidos ósseos e comestíveis e a concentração de gordura corporal, uma vez que a gordura tem baixo teor de minerais e, portanto, dilui esses elementos.

O decréscimo no conteúdo de cálcio e fósforo por unidade de peso, em função do aumento do peso corporal, também foi observado em outras espécies, como bovinos (CARVALHO, 1989; LANA et al., 1992; PIRES et al., 1993; ESTRADA, 1996; e PAULINO, 1996) e caprinos (RESENDE, 1989; RIBEIRO, 1995).

A relação Ca:P encontrada neste trabalho para cordeiros Santa Inês variou de 1,76 a 1,86. Estes resultados estão próximos dos valores de 1,80 e 1,76 a 1,71 preconizados pelo ARC (1980) e AFRC (1991), respectivamente.

A partir de peso vivo, peso corporal vazio e quantidades corporais de cálcio e fósforo dos animais estudados, foram ajustadas equações de regressão para estimar o PCV, em função do PV e, para estimar a quantidade de cálcio e fósforo presentes no corpo vazio, em função do PCV:

$$
\begin{array}{ll}
\text { PCV }(g) \text { PCV } & =-671,20+0,9089 \text { PV } \\
& \mathrm{R}^{2}=96,68 \% \\
\text { Fósforo }(g) \log P & =-0,91433+0,721648 \\
\text { Log PCV } & \mathrm{R}^{2}=92,12 \% \\
\text { Cálcio }(\mathrm{g}) \log \mathrm{Ca} & =-1,04340+0,813209 \\
\text { Log PCV } & \mathrm{R}^{2}=88,61 \%
\end{array}
$$

Os valores dos coeficientes de determinação encontrados para as equações acima foram significativos $(\mathrm{P}<0,01)$ e mostram que as equações foram bem ajustadas, com baixa dispersão dos dados em torno da linha de regressão.

A partir destas equações, estimou-se a composição em cálcio e fósforo do corpo vazio dos animais, em função do peso corporal vazio dos mesmos (Tabela 3 ).

Assim como ocorreu com a composição corporal, os valores estimados da concentração corporal de cálcio e fósforo também diminuíram em função do aumento no peso vivo (Tabela 3). ANNENKOV (1982) também encontrou valores de concentração corporal de cálcio e fósforo decrescentes para cordeiros na faixa de 10 a $30 \mathrm{~kg}$ de PV, sendo que o conteúdo de cálcio variou de 10,30 a 10,20 g Ca/ $/ \mathrm{kg}$ de PCV e o de fósforo, de 6,40 a 5,70 g P/kg de PCV.

Como já discutido anteriormente, este decréscimo na concentração corporal de cálcio e fósforo é atribuído, principalmente, à redução no crescimento ósseo, à medida que aumenta o peso corporal e se aproxima do peso adulto do grupo genético ao qual pertence. 
Tabela 2 - Peso ao abate e composição do corpo vazio para os animais referência, alimentação restrita e ad libitum

Table 2 - Slaughter weight and the empty body composition for the animals reference, restrict and ad libitum fed

\begin{tabular}{|c|c|c|c|}
\hline & $\begin{array}{l}\text { Referência } \\
\text { Reference }\end{array}$ & $\begin{array}{l}\text { Restrita } \\
\text { Restrict }\end{array}$ & Ad Libitum \\
\hline $\begin{array}{l}\text { Peso ao abate }(\mathrm{kg}) \\
\text { Slaughterweight }\end{array}$ & $15,02 \pm 0,614$ & $14,50 \pm 0,774$ & $24,92 \pm 0,664$ \\
\hline $\begin{array}{l}\text { Peso corporal vazio }(\mathrm{kg}) \\
\text { Empty body weight }\end{array}$ & $12,90 \pm 1,143$ & $12,65 \pm 1,367$ & $21,91 \pm 1,384$ \\
\hline $\begin{array}{l}\text { Matéria seca }(\%) \\
\text { Dry matter }\end{array}$ & $32,36 \pm 3,034$ & $34,53 \pm 1,580$ & $35,91 \pm 1227$ \\
\hline $\begin{array}{l}\text { Proteína }(\% \mathrm{MN})^{1} \\
\text { Protein }(\% F M)\end{array}$ & $17,52 \pm 0,736$ & $17,93 \pm 0,738$ & $17,14 \pm 0,942$ \\
\hline $\begin{array}{l}\text { Gordura }(\% \mathrm{MN})^{1} \\
\text { Fat }(\% F M)\end{array}$ & $10,02 \pm 3,084$ & $12,17 \pm 1,506$ & $14,43 \pm 0,985$ \\
\hline $\begin{array}{l}\text { Energia }(\mathrm{kcal} / \mathrm{kg} \mathrm{MN})^{1} \\
\text { Energy }(k c a l / k g \text { FM })\end{array}$ & $2184 \pm 402$ & $2340 \pm 241$ & $2519 \pm 36$ \\
\hline $\begin{array}{l}\mathrm{Ca}(\% \mathrm{MN}[F M])^{1} \\
\mathrm{P}\left(\% \mathrm{MN}_{[F M]}{ }^{1}\right.\end{array}$ & $\begin{array}{l}1,520 \pm 0,062 \\
0,861 \pm 0,044\end{array}$ & $\begin{array}{l}1,589 \pm 0,097 \\
0,899 \pm 0,034\end{array}$ & $\begin{array}{l}1,396 \pm 0,160 \\
0,750 \pm 0,050\end{array}$ \\
\hline
\end{tabular}

${ }^{1}$ Matéria natural MN (Fresh matter [FM]).

Tabela 3 - Concentração de cálcio e fósforo no corpo vazio dos animais

Table 3 - Calcium and phosphorus concentration in the empty body of the animals

\begin{tabular}{lccc}
\hline Peso vivo & $\begin{array}{c}\text { Peso corporal vazio } \\
\text { Body weight } \\
\text { Empty bodyweight }\end{array}$ & \multicolumn{2}{c}{$\begin{array}{c}\text { Mineral (g/kg PCV) } \\
\text { Mineral }(\mathrm{g} / \mathrm{kg} \text { EBW })\end{array}$} \\
\cline { 3 - 4 }$(\mathrm{kg})$ & 12,96 & $\mathrm{Ca}$ & $\mathrm{P}$ \\
\hline 15,0 & 15,23 & 14,431 & 8,728 \\
17,5 & 17,51 & 14,588 & 8,345 \\
20,0 & 19,78 & 14,259 & 8,027 \\
22,5 & 22,05 & 13,973 & 7,759 \\
25,0 & & 11,0 & 6,528 \\
\hline ARC $(1980)$ & & &
\end{tabular}

Entretanto, os resultados encontrados nesta pesquisa diferem dos valores propostos pelo ARC (1980), que considera a deposição de cálcio e fósforo constante, independente do peso do animal, e estima valor de $11 \mathrm{~g}$ Ca e $6 \mathrm{~g} \mathrm{P} / \mathrm{kg}$ de PCV. Porém, em 1991, o AFRC reexaminou o modelo proposto pelo ARC (1980) e admitiu que o depósito de cálcio e fósforo decresce com a maturidade, o que confirma os resultados encontrados nesta pesquisa.

Estas diferenças nas concentrações de cálcio e fósforo corporais são reflexo principalmente das diferenças existentes na proporção de ossos na carcaça, uma vez que $99 \%$ do conteúdo de cálcio e $80 \%$ do conteúdo de fósforo no corpo estão nos ossos. Outro fator que explica essas diferenças nas concentrações desses minerais é a variação na concentração de gordura, a qual é função de idade, raça, grupo genético, sexo, manejo alimentar e condições climáticas ao qual o animal é submetido.

A partir da derivação das equações de predição da composição corporal de cálcio e fósforo, foram obtidas as equações que permitiram estimar a quantidade de cálcio e fósforo depositada por quilograma de ganho em PCV:

Fósforo $(\mathrm{g}) \quad \mathrm{Y}^{\prime}=0,087901 . \mathrm{PCV}^{-0,278352}$

Cálcio $(\mathrm{g}) \quad \mathrm{Y}^{\prime}=0,078679 . \mathrm{PCV}^{-0,186791}$

A concentração de cálcio no ganho de PCV encontrada nesta pesquisa variou de 12,55 a $11,36 \mathrm{~g} / \mathrm{kg}$ PCV e o fósforo, de 6,30 a $5,43 \mathrm{~g} / \mathrm{kg}$ PCV, para animais com 12,96 a $22,05 \mathrm{~kg}$ de PCV, respectivamente.

Visto que as quantidades de cálcio e fósforo no ganho de PCV são reflexo da composição corporal, os valores encontrados nesta pesquisa para a composição do ganho diferiram dos valores preconizados pelo ARC (1980) e NRC (1985).

Para a composição do ganho, o AFRC (1991) reexaminou e modificou o modelo proposto pelo ARC (1980), introduzindo o peso à maturidade no cálculo e considerando, portanto, o decréscimo na deposição destes minerais, com o avanço da maturidade. Para animais com $15 \mathrm{~kg} \mathrm{PV}$, os valores encontrados por este comitê foram 11,48 g Ca/kg PV e 6,62 g P/kg PV.

Da mesma forma que para a composição corporal, essas diferenças na quantidade de cálcio e fósforo do ganho são reflexo, principalmente, das diferenças existentes na proporção de ossos e gordura na carcaça. Portanto, valores sobre a composição de ganho, obtidos com raças e condições climáticas diferentes, devem ser utilizados com cautela (SILVA, 1996).

Os coeficientes de absorção e as exigências 
líquidas para mantença foram estimados, a partir de equações de regressão, correlacionando a quantidade ingerida (g/dia) pelos animais e a quantidade retida (g/dia) no corpo vazio dos mesmos:

Fósforo $(\mathrm{g} / \mathrm{dia}) \mathrm{P}$ retido $=-0,325+0,5528 \mathrm{P}$ ingerido $\mathrm{R}^{2}=88,61$

Cálcio $(\mathrm{g} / \mathrm{dia})$ Ca retido $=0,305+0,4423 \mathrm{Ca}$ ingerido $\mathrm{R}^{2}=44,23$

Os valores dos coeficientes de determinação encontrados para as equações acima foram significativos $(\mathrm{P}<0,05)$ e mostram que as equações foram bem ajustadas, com baixa dispersão dos dados em torno da linha de regressão.

As exigências líquidas de mantença para cálcio e fósforo, encontradas neste trabalho para animais entre 15 e $25 \mathrm{~kg}$ de peso vivo, foram $305 \mathrm{mg} \mathrm{Ca} / \mathrm{dia}$ e $325 \mathrm{mg} \mathrm{P} / \mathrm{dia}$. Os valores de exigência líquida de mantença de cálcio deste trabalho, para cordeiros com $25 \mathrm{~kg}$, foram maiores que os obtidos por OKOYE et al. (1980), 227,50 mg Ca/dia, e HODGE (1973), 220,00 mg Ca/dia, porém encontram-se dentro da faixa de valores estimados pelo ARC (1980), que varia de 240 a $400 \mathrm{mg} \mathrm{Ca/dia} \mathrm{para} \mathrm{cordeiros} \mathrm{com} 15$ a $25 \mathrm{~kg} \mathrm{PV}$, respectivamente. É importante ressaltar que estas diferenças ocorrem porque, nos estudos realizados, as condições foram diferentes entre si e principalmente as raças utilizadas foram outras.

O coeficiente de absorção encontrado neste trabalho para o cálcio e fósforo foi, respectivamente, $0,44 \mathrm{e}$ 0,55 . Estes valores foram inferiores aos preconizados pelo ARC (1980), 0,68 para o cálcio e 0,73 para o fósforo, e NRC (1985), 0,60 para o cálcio e 0,70 para o fósforo, porém foram semelhantes aos encontrado por OKOYE et al. (1980), 0,55 para o cálcio e 0,57 para o fósforo. Estas diferenças entre os valores dos coeficientes de absorção do cálcio e fósforo podem ser reflexo das diferenças entre os alimentos utilizados, assim como dos efeitos das condições experimentais e

Tabela 4 - Concentração de cálcio e fósforo por kg de ganho de peso corporal vazio

Table 4 - Calcium and phosphorus concentration per $\mathrm{kg}$ of empty body weight gain

\begin{tabular}{|c|c|c|c|}
\hline \multirow{2}{*}{$\begin{array}{l}\text { Peso vivo } \\
\text { Bodyweight } \\
(\mathrm{kg})\end{array}$} & \multirow{2}{*}{$\begin{array}{l}\text { Peso corporal vazio } \\
\text { Empty body weight } \\
(\mathrm{kg})\end{array}$} & \multicolumn{2}{|c|}{$\begin{array}{c}\text { Mineral }(\mathrm{g} / \mathrm{kg} \text { PCV }) \\
\text { Mineral }(g / k g E B W)\end{array}$} \\
\hline & & $\mathrm{Ca}$ & $\mathrm{P}$ \\
\hline 15 & 12,96 & 12,548 & 6,298 \\
\hline 17,5 & 15,23 & 12,176 & 6,022 \\
\hline 20,0 & 17,51 & 11,863 & 5,793 \\
\hline 22,5 & 19,78 & 11,596 & 5,599 \\
\hline 25,0 & 22,05 & 11,363 & 5,432 \\
\hline $\operatorname{ARC}(1980)$ & & 11,0 & 6,0 \\
\hline
\end{tabular}

das diferenças entre as raças utilizadas.

As exigências líquidas de cálcio e fósforo para o ganho de peso vivo foram calculadas dividindo-se as exigências líquidas para o ganho de peso corporal vazio (Tabela 4) pelo fator 1,10, calculado a partir das equações de conversão de PCV para PV. As exigências líquidas e dietéticas de cálcio e fósforo para cordeiros Santa Inês são apresentadas nas Tabelas 5 a 8 .

Fazendo um paralelo entre as exigências líquidas para o ganho de cálcio obtidas neste trabalho e àquelas citadas pelo ARC (1980), observa-se que estas são, aproximadamente, $3,73 \%$ superiores para cordeiros com $15 \mathrm{~kg}$ de peso vivo e $9,39 \%$ inferiores para animais com $25 \mathrm{~kg}$.

O aumento da diferença nas exigências de cálcio para o ganho, à medida que aumenta o peso vivo dos animais, é atribuído ao fato de o ARC (1980) considerar a concentração de cálcio no ganho de peso vivo constante, durante o crescimento do animal. Entretanto, neste trabalho, considera-se que ocorre diminuição na quantidade de cálcio por unidade de ganho de peso vivo, à medida que aumenta o peso do animal.

As exigências dietéticas totais de cálcio obtidas neste trabalho são, aproximadamente, $24,45 \%$ superiores às citadas pelo NRC (1985), para cordeiros com $15 \mathrm{~kg}$ de peso vivo, e 11,42\% inferiores para animais com $25 \mathrm{~kg}$. Por outro lado, com relação às normas preconizadas pelo AFRC (1991), as exigências dietéticas de cálcio encontradas neste trabalho foram 42,31 e $33,97 \%$ superiores para animais com 15 e $25 \mathrm{~kg}$ de PV, respectivamente, apresentando taxa de ganho de $200 \mathrm{~g} / \mathrm{dia}$.

Já no caso do fósforo, as exigências líquidas para o ganho obtidas neste trabalho são, aproximadamente, 4,67\% inferiores às citadas pelo ARC (1980), para cordeiros com $15 \mathrm{~kg}$ de peso vivo, e 17,67\% inferiores para animais com $25 \mathrm{~kg}$.

Para animais com $25 \mathrm{~kg}$ de peso vivo e taxa de ganho diário de 200 g, ANNENKOV (1982) recomenda ingestão diária de fósforo $2,45 \mathrm{~g}$; o NRC (1985), 2,85; e o ARC (1980), 2,00 g. Esses valores são, respectivamente, 3,11 e $19,95 \%$ superiores e $18,80 \%$ inferior aos encontrados neste trabalho.

Nota-se que existe diferença entre os valores deste trabalho e os citados pela literatura. Segundo SILVA (1995), as estimativas das exigências líquidas de $\mathrm{Ca}$ e $\mathrm{P}$ de diversos trabalhos conduzidos com bovinos, no Brasil, diferiram em cerca de $100 \%$ dos valores propostos pelo AFRC (1991). Possivelmente, as diferenças nas estimativas das exigências líquidas e dietéticas de cálcio e fósforo deste trabalho, com- 
Rev. bras. zootec.

Tabela 5 - Estimativas das exigências líquidas de cálcio para o ganho em peso vivo

Table 5 - Estimates of the net requirements of calcium for live weight gain

\begin{tabular}{cccc}
\hline \multirow{2}{*}{$\begin{array}{c}\text { Peso vivo } \\
\text { Live weight } \\
(\mathrm{kg})\end{array}$} & \multicolumn{3}{c}{$\begin{array}{c}\text { Ganho em peso vivo (g/dia) } \\
\text { Live weight gain }(\mathrm{g} / \text { day) }\end{array}$} \\
\cline { 2 - 4 } & 100 & 200 & 300 \\
\hline 15 & 1,141 & 2,282 & 3,423 \\
20 & 1,078 & 2,156 & 3,234 \\
25 & 1,033 & 2,066 & 3,099 \\
\hline
\end{tabular}

Tabela 6 - Estimativas das exigências dietéticas de cálcio para o ganho em peso vivo

Table 6 - Estimates of the dietary requirements of calcium for live weight gain

\begin{tabular}{cccc}
\hline $\begin{array}{c}\text { Peso vivo } \\
\text { Live weight } \\
(\mathrm{kg})\end{array}$ & \multicolumn{3}{c}{$\begin{array}{c}\text { Ganho em peso vivo (g/dia) } \\
\text { Live weight gain }(\mathrm{g} / \text { day) }\end{array}$} \\
\cline { 2 - 4 } & 100 & 200 & 300 \\
\hline 15 & 2,580 & 5,160 & 7,740 \\
20 & 2,437 & 4,874 & 7,311 \\
25 & 2,335 & 4,670 & 7,005 \\
\hline
\end{tabular}

paradas às do ARC (1980), NRC (1985) e AFRC (1991), são reflexo das diferenças existentes na composição corporal dos animais estudados.

Como discutido anteriormente, a composição corporal e, conseqüentemente, as exigências de cálcio e fósforo variam, principalmente, em função da proporção de ossos na carcaça e concentração de gordura, que, por sua vez, são influenciadas por idade do animal, raça, grupo genético, sexo, manejo alimentar e condições climáticas. Portanto, os valores de exigências para ovinos preconizados pelo ARC (1980), NRC (1985) e AFRC (1991) devem ser utilizados com certa cautela, uma vez que estes resultados foram obtidos a partir de raças, condições climáticas e alimentação diferentes.

\section{Conclusões}

Os valores de composição corporal de cálcio estimados por este trabalho variaram de 15,431 a $13,973 \mathrm{~g} / \mathrm{kg}$ de peso corporal vazio e os de fósforo, de 8,728 a $7,528 \mathrm{~g} / \mathrm{kg}$ de peso corporal vazio.

Os requerimentos líquidos de cálcio e fósforo para a mantença, estimados para animais entre 15 e $25 \mathrm{~kg}$ de peso vivo, foram $305 \mathrm{mg}$ de Ca/dia e $325 \mathrm{mg}$ de $\mathrm{P} /$ dia. Os coeficientes de absorção de cálcio e fósforo encontrados foram 0,44 e 0,55 , respectivamente.

Os requerimentos líquidos de cálcio e fósforo
Tabela 7 - Estimativas das exigências líquidas de fósforo para o ganho em peso vivo

Table 7 - Estimates of the net requirements of phosphorus for live weight gain

\begin{tabular}{cccc}
\hline \multirow{2}{*}{$\begin{array}{c}\text { Peso vive weight } \\
\text { Live }\end{array}$} & \multicolumn{3}{c}{$\begin{array}{c}\text { Ganho em peso vivo (g/dia) } \\
\text { Live weight gain }(\mathrm{g} / \text { day })\end{array}$} \\
\cline { 2 - 4 } & 100 & 200 & 300 \\
\hline 15 & 0,572 & 1,144 & 1,716 \\
20 & 0,527 & 1,054 & 1,581 \\
25 & 0,494 & 0,988 & 1,482 \\
\hline
\end{tabular}

Tabela 8 - Estimativas das exigências dietéticas de fósforo para o ganho em peso vivo

Table 8 - Estimates of the dietary requirements of phosphorus for live weight gain

\begin{tabular}{cccc}
\hline $\begin{array}{c}\text { Peso vivo } \\
\text { Live weight } \\
(\mathrm{kg})\end{array}$ & \multicolumn{3}{c}{$\begin{array}{c}\text { Ganho em peso vivo (g/dia) } \\
\text { Live weight gain (g/day) }\end{array}$} \\
\cline { 2 - 4 } & 100 & 200 & 300 \\
\hline 15 & 1,035 & 2,070 & 3,105 \\
20 & 0,953 & 1,906 & 2,859 \\
25 & 0,894 & 1,788 & 2,682 \\
\hline
\end{tabular}

para o ganho em peso estimados variaram de $11,41 \mathrm{a}$ $10,33 \mathrm{~g} / \mathrm{kg}$ de peso vivo e os de fósforo, de $5,72 \mathrm{a}$ $4,94 \mathrm{~g} / \mathrm{kg}$ de peso vivo.

A composição corporal e as exigências líquidas de cálcio e fósforo, estimadas por intermédio de tabelas desenvolvidas com raças e condições climáticas diferentes, não refletiram a real composição corporal de cordeiros Santa Inês criados na região Sudeste do Brasil.

\section{Referências Bibliográficas}

AGRICULTURAL AND FOOD RESEARCH COUNCIL AFRC. 1991. A reappraisal of the calcium and phosphorus requirements of sheep and cattle. Report 6, Nutrition Abstract review, series B., 61(9):573-612.

AGRICULTURAL RESEARCH COUNCIL - ARC. 1980. The nutrient requirements of farm livestock. London. 351p.

ANNENKOV, B.N. 1982. Mineral feeding of sheep. In: GEORGIEVSKII, V.I., ANNENKOV, B.N., SAMOKHIN, V.I. (Eds) Mineral nutrition of animals. London: Butterworths. p.321-354.

BOIN, C. Exigências de minerais pelas categorias do rebanho bovino e funções desses nutrientes. In: SIMPÓSIO SOBRE NUTRIÇÃO DE BOVINOS, 3, Piracicaba, 1985. Anais... Piracicaba: FEALQ, 1985, p.15.

BURTON, J.H., ANDERSON, M., REID, J.T. 1974. Some biological aspects of partial starvation. The effect of weight loss and regrowth on body composition in sheep. Br. J. Nut., 32:515-527.

BURTON, J.H., REID, J.T. 1969. Interrelationships among energy input, body size, age and body composition of sheep. J. Nut., 97:517-524. 
CARVALHO, D.R. Composição corporal e exigências nutricionais de macroelementos inorgânicos de bovinos. Viçosa: UFV, 1989. 84p. Dissertação (Mestrado em Zootecnia) - Universidade Federal de Viçosa, 1989.

CAVALHEIRO, A.C.L, TRINDADE, D.S. 1992. Os minerais para bovinos e ovinos criados em pastejo. Porto Alegre: Sagra-DC Luzzato. 141p.

ESTRADA, L.H.C. Composição corporal e exigências de proteína, energia e macroelementos minerais $(\mathrm{Ca}, \mathrm{P}, \mathrm{Mg}, \mathrm{Ke}$ $\mathrm{Na}$ ), características de carcaça e desempenho do nelore $e$ mestiço em confinamento. Viçosa: UFV, 1996. 128p. Tese (Doutorado em Zootecnia) - Universidade Federal de Viçosa, 1996.

GRACE, N.D. 1983. Amounts and distribution of mineral elements associated with fleece-free empty body weight gains in the grazing sheep. New Zealand J. Agric. Res., 26:5970.

HODGE, R.W. 1973. Calcium requirements of the young lamb. II Estimation of calcium requirements by the factorial method. Austr. J. Agric. Res., 24:237-43.

KELLAWAY, R.C. 1973. The effects of plane nutrition, genotype and Sex on growth, body composition and wool production in grazing sheep. J. Agric. Sci., 80:17-27.

LANA, R.P., FONTES, C.A.A., PERON, A.J. et al. 1992. Composição corporal e do ganho de peso e exigências de energia, proteína e macroelementos minerais $(\mathrm{Ca}, \mathrm{P}, \mathrm{Mg}, \mathrm{Na}$ e K) de novilhos de cinco grupos raciais. 3. Conteúdo corporal e do ganho de peso e exigências de macroelementos minerais. R. Soc. Bras. Zootec., 21(03):538-544.

NATIONAL RESEARCH COUNCIL - NRC. 1985. Nutrient requirements of domestic animals: Nutrient requirements of sheep. Washington. 99p.

NOUR, A.Y.M., THONNEY, M.L. 1987. Carcass, soft tissue and bone composition of early and late maturing steers fed two diets in two housing types and serially slaughtered a wide weight range. J. Agric. Sci., 109:345-355.

OKOYE, F.C., UMUNNA, N.N., CHINEME, C.N. 1980. Calcium and phosphorus requirements of growing yankasa lambs in the savanna region of Nigeria. Estimation of calcium and phosphorus requirements by the factorial method. East African Agric. For. J., 45(4):269-76.

PAULINO, M.F. Composição corporal e exigências de energia e macroelementos minerias ( $\mathrm{Ca}, \mathrm{P}, \mathrm{Mg}$, Na e K) de bovinos não castrados de quatro raças zebuínas em confinamento. Viçosa: UFV, 1996, 80p. Tese (Doutorado em Zootecnia) Universidade Federal de Viçosa, 1996.
PIRES, C.C., FONTES, C.A.A., GALVÃO, J.G. et al. 1993. Exigências nutricionais de bovinos de corte em acabamento. III - Exigências de cálcio e fósforo para o ganho. R. Soc. Bras. Zootec., 22(01):133-143.

RATTRAY, P.V., GARRET, W.N., MEYER, H.H. et al. 1973. Body and carcass composition of targhee and finn-targhee lambs. J. Anim. Sci., 37(4):892-897.

RESENDE, K.T. Métodos de estimativa da composição corporal $e$ exigências nutricionais de proteína, energia $e$ macroelementos inorgânicos de caprinos em crescimento. Viçosa: UFV, 1989. 130p. Tese (Doutorado em Zootecnia) - Universidade Federal de Viçosa, 1989.

RIBEIRO, S.D.A. Composição corporal e exigências em proteína, energia e macrominerais de caprinos mestiços em fase inicial de crescimento. Jaboticabal: FCAVJ/UNESP, 1995. 100p. Dissertação (Mestrado em Zootecnia) - Universidade Estadual Paulista, 1995.

SEARLE, T.W., GRAHAM, N.M., SMITH, E. 1979. Studies of weaned lambs, before, during and after a period of weight loss. II Body composition. Austr. J. Agric. Res.., 30:525-531.

SEARLE, T.W., GRAHAM, N.M., DONNELLY, J.B. 1982. The effect of plane of nutrition on the body composition of two breeds of weaner sheep fed a high protein diet. J. Agric. Sci., 98:241-245.

SILVA, D.J. 1981. Análise de alimentos (Métodos químicos e biológicos). Viçosa: UFV, Impr. Univers. 165p.

SILVA, J.F.C. Exigências de macroelementos inorgânicos para bovinos : o sistema ARC/AFRC e a experiência no Brasil. In: Simpósio internacional sobre exigências nutricionais de ruminantes, Viçosa, 1995. Anais...Viçosa, 1995, p. 467-504.

SILVA, J.F.C. 1996. Metodologia para determinação de exigências nutricionais de ovinos. In: SILVA SOBRINHO, A.G., BATISTA, A.M.V., SIQUEIRA, E.R. et al. (Eds.) Nutrição de ovinos. Jaboticabal: FUNEP. p.1-68

Recebido em: 29/12/98

Aceito em: 30/06/99 